\title{
An exploration of genetic health professionals' experience with direct-to-consumer genetic testing in their clinical practice
}

\author{
Gemma R Brett $^{1,2}$, Sylvia A Metcalfe ${ }^{1,2}$, David J Amor ${ }^{1,2}$ and Jane L Halliday ${ }^{\star, 1,2}$
}

Direct-to-consumer genetic testing (DTC-GT) allows individuals to obtain genetic tests directly from companies without necessarily involving health professionals. This study explores genetic health professionals' opinions of health-related DTC-GT and the reported frequency of individuals presenting to clinical genetics services after undertaking testing. Genetic counsellors and clinical geneticists, members of the Human Genetics Society of Australasia, completed an online survey in mid 2011. The 130 genetic counsellors (estimated response fraction $=43 \%$ ) and 38 clinical geneticists (estimated response fraction $=46 \%$ ) had mixed opinions regarding DTC-GT, with only $7 \%$ confident in accurately interpreting and explaining DTC-GT results. Nineteen respondents $(11 \%)$ reported one or more client(s) referred to them after undertaking DTC-GT. Descriptions of 25 clients were extracted from responses, and respondents reported that all clients were concerned for the health of either themselves or family members. Most clients presented to genetic clinics specifically as a result of their DTC-GT (96\%) and were self or GP referred (92\%). Respondents perceived that their clients typically undertook DTC-GT because they wanted to identify monogenic conditions, including carrier testing and/or know their susceptibility or predisposition for complex conditions (88\%). The majority of clients needed help interpreting DTC-GT results $(80 \%)$, however in general were not questioning the validity of their DTC-GT results $(92 \%)$ nor seeking further genetic testing $(84 \%)$. Currently, DTC-GT is not a major reason for referral to clinical genetics services in Australia and New Zealand and the majority of genetic health professionals lack confidence in being able to accurately interpret and explain DTC-GT results.

European Journal of Human Genetics (2012) 20, 825-830; doi:10.1038/ejhg.2012.13; published online 8 February 2012

Keywords: direct-to-consumer; health professional; genetic testing; genetic counsellor; attitudes

\section{INTRODUCTION}

Many direct-to-consumer genetic tests (DTC-GT) have unknown clinical validity and utility, ${ }^{1-3}$ and their use to assess disease risk is therefore controversial. In addition, the personalised genetic risk information provided by DTC-GT may be interpreted solely by consumers without guidance from appropriate health professionals. For example, in a recent longitudinal cohort study to measure the effects of DTC-GT, only $10.4 \%$ of subjects reported discussing their results with one of the genetic counsellors provided by the testing company. ${ }^{4}$ Un-informed interpretation of genetic test results may inappropriately heighten health-related anxiety or apathy and subsequently lead to poor life choices; however, this has not been proven. There is also potential for other psychological effects on consumers due to lack of knowledge and support. The long-term demands on health services and impact on health behaviour as a result of DTC-GT are yet to be established.

Various publications have identified a number of other issues with ethical, legal and psychological implications such as a lack of informed consent, a lack of confidentiality and the potential for testing in minors. ${ }^{5-9}$ First, informed consent may be compromised by lack of provision of appropriate facts. ${ }^{9}$ Second, confidentiality may be breached through integration of online social networking opportunities into the websites of DTC-GT companies which promote sharing of personal genomic information ${ }^{7}$ and, although this may facilitate collaboration between clients and researchers, the consequences of these potential confidentiality breaches are difficult to predict. ${ }^{8}$ Third, although most DTC-GT companies maintain that their services are not intended to attract minors, testing in minors continues to occur. ${ }^{5,6}$

Although there is a body of literature regarding DTC-GT, observational studies are limited. Some case studies have reported DTC-GT results with apparent clinical utility ${ }^{10-12}$ and other studies report results with no clinical utility. ${ }^{13-15}$ The perceptions and attitudes of the general public towards DTC-GT have also been explored. Although a strong public interest in predictive genetic testing has been reported, ${ }^{16-19}$ this is often accompanied by the desire for closer involvement of a medical professional. ${ }^{16,18,19} \mathrm{~A}$ number of studies have examined the knowledge and experience of genetic health professionals ${ }^{20-22}$ and other health professionals $\mathrm{s}^{20,22-24}$ with DTC-GT. The majority of these studies surveyed health professionals in the USA ${ }^{20,21,23}$ with one study sampling another area, the Kanagawa Prefecture of Japan..$^{22}$ Response fractions varied, with one study reporting a fraction as low as $3.3 \%, 20$ and another as high as $36 \%{ }^{21}$ To date, there is no published literature regarding the experience of health professionals in Australasia.

${ }^{1}$ Murdoch Childrens Research Institute, Royal Children's Hospital, Parkville, Victoria, Australia; ${ }^{2}$ Department of Paediatrics, The University of Melbourne, Parkville, Victoria, Australia

${ }^{*}$ Correspondence: Associate Professor JL Halliday, Murdoch Childrens Research Institute, 10th Floor, Royal Children's Hospital, Flemington Road, Parkville, Victoria 3052, Australia. Tel: +61 38341 6360; Fax: +61 38341 6212; E-mail: janehalliday.h@mcri.edu.au

Received 14 September 2011; revised 22 December 2011; accepted 6 January 2012; published online 8 February 2012 
As there are currently no major providers of health-related DTC-GT based in Australasia, individuals in this region who wish to undertake DTC-GT must send their samples to international laboratories. Although studies describing the experiences of consumers in the USA are emerging, ${ }^{4,25}$ little is known about the experience of individuals in other countries. There are additional considerations for these individuals that are not present for those residing in the USA, such as difficulties accessing telephone counselling services provided by some DTC-GT companies. The global uptake of DTC-GT is currently unknown as publically available information regarding DTC-GT purchase rates is limited. ${ }^{26}$ Therefore, it is difficult to assess the extent to which DTC-GT is impacting clinical genetics services at an international level. The research described here responds to a gap in existing scientific knowledge and provides insight into the experiences of genetic health professionals within the countries that do not currently have local health-related DTC-GT companies.

The aims of this study were to determine the reported frequency of individuals presenting to genetics services after undertaking DTC-GT, to explore the opinions and experiences of genetic health professionals regarding DTC-GT and to explore the perceived opinions and experiences of clients who have undertaken DTC-GT.

\section{METHODS}

A novel online survey comprising 20 questions was made available to genetic health professionals who are members of the Human Genetics Society of Australasia (HGSA), the Australasian forum for the various disciplines within the field of human genetics. Questions were drafted after reviewing the existing literature and consensus regarding the relevance and phrasing of questions was achieved amongst the authors after several iterations. The survey was piloted with Master of Genetic Counselling students, followed by further modifications by the authors. Ethics approval was obtained from the Human Research Ethics Committee at the University of Melbourne. All survey responses were voluntary, confidential and anonymous. Respondents were advised that completion of the survey would imply consent to participate.

The opening question of the survey established respondents' professional status and therefore their eligibility for this study (see Supplementary Information for a text form of the online survey). Genetic health professionals in Australasia include genetic counsellors, associate genetic counsellors (who have attained the relevant academic qualification but not yet completed their certification requirements), clinical geneticists and clinical genetics trainees. The remainder of the survey explored their: (1) opinions regarding DTC-GT use; and (2) experiences with clients who had undertaken DTC-GT. Instructions stated that the survey was concerned with health-related DTCGT that could also be offered in a clinical setting (therefore, not paternity testing, ancestry testing, nutrigenomics, etc). Only those respondents who reported having experience with such DTC-GT in their clinical practice answered both parts of the survey. The survey took approximately 10-15 min to complete.

The survey was hosted on www.surveymonkey.com and invitations to participate were distributed by email to HGSA members in mid April 2011, with periodic reminder emails in the following months. The number of members registered with two relevant HGSA special interest groups was used to determine the response fraction: the Australasian Society of Genetic Counsellors (ASGC) $(n=299)$ and the Australasian Association of Clinical Geneticists (AACG) $(n=82)$.

Analysis of Part 1 data was performed using StataIC, version 11 (StataCorp LP, College Station, TX, USA). For the initial analysis, respondents were grouped by profession ('genetic counsellors' including associate genetic counsellors and 'clinical geneticists' including clinical genetics trainees) and $\chi^{2}$ tests performed to identify any significant differences in opinion between professions. In order to examine the views of all respondents who expressed a firm opinion regarding DTC-GT, responses from both professions were combined. The response 'possibly' was then excluded and $\chi^{2}$ tests performed to identify relationships between firm opinions. $P$ values $\leq 0.05$ were considered signifi- cant. Details of each client reported in Part 2 were extracted for analysis in Microsoft Office Excel 2007 and descriptive statistics were used to summarise client characteristics.

\section{RESULTS}

A total of 218 respondents attempted the survey, of whom 168 were genetic health professionals and were therefore eligible to proceed beyond the first question. An overall response fraction of $44 \%$ was achieved, with 130 genetic counsellors (response fraction=43\%) and 38 clinical geneticists (response fraction $=46 \%$ ) completing Part 1 of the survey. Overall, 82 percent of respondents reported they were currently practising in their listed profession $(n=138)$.

\section{Genetic health professionals' opinions regarding DTC-GT}

Many respondents selected 'possibly' in answer to opinion-related questions, rather than expressing a firm opinion, as shown in Table 1. The majority of respondents do not consider DTC-GT useful for individuals who want anonymous testing $(n=83,54 \%)$, are driven by curiosity $(n=83,54 \%)$ or are geographically isolated $(n=93,60 \%)$. Note that respondents may not have answered every question and therefore the total number of responses per question varies in both Tables 1 and 2. Genetic counsellors were more likely than clinical geneticists to be of the firm opinion that DTC-GT is useful both for individuals who want anonymous testing (eg for insurance purposes) $(P=0.02)$ and those who are geographically isolated $(P=0.04)$. There is also weak evidence that genetic counsellors were more likely than clinical geneticists to be of the firm opinion that DTC-GT is useful for individuals who are driven by curiosity $(P=0.07)$. No other significant differences in opinion were observed between professions.

Only $7 \%$ of respondents were confident in accurately interpreting and explaining DTC-GT results. Respondents who reported they would consider undertaking DTC-GT themselves $(n=14)$ expressed firm opinions in other areas; they were more likely to consider DTC-GT useful for individuals who want anonymous testing $(P<0.001)$, are driven by curiosity $(P<0.001)$ or are geographically isolated $(P=0.001)$ and all felt confident in accurately interpreting and explaining DTC-GT results $(P<0.001)$. Similarly, respondents who reported they were confident in accurately interpreting and explaining DTC-GT results $(n=11)$ expressed firm opinions in other areas; they were more likely to consider DTC-GT useful for individuals who want anonymous testing $(P=0.03)$, are driven by curiosity $(P=0.005)$ or are geographically isolated $(P=0.002)$.

\section{DTC-GT in genetic health professionals' clinical practice}

A total of 19 respondents (11\%), 15 genetic counsellors (12\%) and 4 clinical geneticists $(11 \%)$, reported having had one or more clients consult with them after undertaking DTC-GT. Four of these 19 respondents $(21 \%)$ who reported experience with DTC-GT in their clinical practice would consider undertaking DTC-GT themselves (see Table 2), compared with $9 \%$ of all respondents. There is a slight shift to more confident opinions amongst those respondents with DTC-GT experience, when compared with the opinions those respondents with no DTC-GT experience (see Table 2). No other differences were noted between those who had experience with DTC-GT and those who did not.

Eighteen of the respondents who reported experience with DTC-GT in their clinical practice went on to complete Part 2 of the survey, from which 25 client descriptions were extracted. Tables 3 and 4 summarise the responses from these 18 respondents. Characteristics of the clients were examined to confirm they met the definition of DTC-GT and ensure there were no instances of the same client being reported by 


\begin{tabular}{|c|c|c|c|c|c|c|c|c|c|}
\hline & \multicolumn{3}{|c|}{$\begin{array}{c}\text { Genetic counsellors, } n=130 \\
n(\%)\end{array}$} & \multicolumn{3}{|c|}{$\begin{array}{c}\text { Clinical geneticists, } \mathrm{n}=38 \\
\mathrm{n}(\%)\end{array}$} & \multicolumn{3}{|c|}{$\begin{array}{l}\text { Total } \\
\mathrm{n}(\%)\end{array}$} \\
\hline & Yes & Possibly & No & Yes & Possibly & No & Yes & Possibly & No \\
\hline \multicolumn{10}{|l|}{ Consider DTC-GT useful for individuals who: } \\
\hline Want anonymous testing & $13(10)$ & $52(42)$ & $59(48)$ & $0(0)$ & $7(23)$ & $24(77)$ & $13(8)$ & $59(38)$ & $83(54)$ \\
\hline Are driven by curiosity & $17(13)$ & $45(36)$ & $62(50)$ & $1(3)$ & $9(29)$ & $21(68)$ & $18(12)$ & $54(35)$ & $83(54)$ \\
\hline Are geographically isolated & $11(9)$ & $46(37)$ & $67(54)$ & $0(0)$ & $5(16)$ & $26(84)$ & $11(7)$ & $51(33)$ & $93(60)$ \\
\hline Are provided appropriate genetic counselling & $66(53)$ & $50(40)$ & $8(6)$ & $8(26)$ & $21(68)$ & $2(6)$ & $74(48)$ & $71(46)$ & $10(6)$ \\
\hline Would consider undertaking DTC-GT themselves & $11(9)$ & $37(30)$ & $76(61)$ & $3(10)$ & $9(29)$ & $19(61)$ & $14(9)$ & $46(30)$ & $95(61)$ \\
\hline Are confident in accurately interpreting and explaining DTC-GT results & $7(6)$ & $63(51)$ & $54(44)$ & $4(13)$ & $17(55)$ & $10(32)$ & $11(7)$ & $80(52)$ & $64(41)$ \\
\hline
\end{tabular}

Abbreviation: DTC-GT, direct-to-consumer genetic testing.

Table 2 Opinions of genetic health professionals with DTC-GT experience and with no DTC-GT experience

\begin{tabular}{|c|c|c|c|c|c|c|}
\hline & \multirow{2}{*}{\multicolumn{3}{|c|}{$\begin{array}{l}\text { With experience }(\mathrm{n}=19) \\
\mathrm{n}(\%)\end{array}$}} & \multirow{2}{*}{\multicolumn{3}{|c|}{$\begin{array}{l}\text { With no experience }(\mathrm{n}=149) \\
\mathrm{n}(\%)\end{array}$}} \\
\hline & & & & & & \\
\hline & Yes & Possibly & No & Yes & Possibly & No \\
\hline \multicolumn{7}{|l|}{ Consider DTC-GT useful for individuals who: } \\
\hline Want anonymous testing & $3(16)$ & $7(37)$ & $9(47)$ & $10(7)$ & $52(38)$ & $74(54)$ \\
\hline Are driven by curiosity & $2(11)$ & $6(32)$ & $11(58)$ & $16(12)$ & $48(35)$ & $72(53)$ \\
\hline Are geographically isolated & $3(16)$ & $3(16)$ & $13(68)$ & $8(6)$ & $48(35)$ & $80(59)$ \\
\hline Are provided appropriate genetic counselling & $10(53)$ & 7 (37) & $2(11)$ & $64(47)$ & $64(47)$ & $8(6)$ \\
\hline Would consider undertaking DTC-GT themselves & $4(21)$ & $5(26)$ & $10(53)$ & $10(7)$ & $41(30)$ & $85(63)$ \\
\hline Are confident in accurately interpreting and explaining DTC-GT results & $3(16)$ & $11(58)$ & $5(26)$ & $8(6)$ & $69(51)$ & $59(43)$ \\
\hline
\end{tabular}

Abbreviation: DTC-GT, direct-to-consumer genetic testing.

two or more respondents. One consultation was reported to have occurred before 2008, 6 during 2009, 12 during 2010 and 6 during the first half of 2011.

Most clients presented to genetic clinics specifically as a result of their DTC-GT $(n=24,96 \%)$ and either referred themselves or were referred by a general practitioner $(n=23,92 \%)$. The one client who was not referred specifically as a result of their DTC-GT was referred by a general practitioner, however, no further information was provided regarding the referral reason. Overall, $80 \%$ of clients had also consulted with non-genetic health professionals in regards to their DTC-GT results $(n=20)$. In all, $48 \%$ of clients $(n=12)$ utilised one of two major DTC-GT companies based in the USA: 23andMe and Counsyl. Other companies reportedly used by clients include deCODEme, Genetic Technologies, GKnowmix, Navigenics and Pathway Genomics. The reported amount paid by clients for their DTC-GT varied considerably: three paid $<\$ 500$, two paid $\$ 500-1000$, three paid \$1500-2000 and six paid $>\$ 2000$ (in USD). The limited data obtained did not show any clear trends between the company used, type of test and amount paid.

Table 3 lists the experiences of clients who consulted with a genetic health professional after undertaking DTC-GT, as reported by the genetic health professional themselves (based on tick box responses). All clients were concerned for their own health $(n=10,40 \%)$ and/or the health of family members $(n=15,60 \%)$, as respondents selected at least one of these characteristics in each case $(n=25,100 \%)$. Typically, clients wanted to identify monogenic conditions, including carrier testing $(n=9,36 \%)$, and/or know their susceptibility or predisposition for complex conditions $(n=19,56 \%)$, as respondents selected at least one of these characteristics in the majority of cases $(n=22,88 \%)$. No clients were reported to have undertaken DTC-GT for pharmacogenetic use.

Respondents' experience(s) with DTC-GT in their clinical practice are described in Table 4 . Only $28 \%$ of initial counselling sessions resulted in no further action on behalf of the client $(n=7)$, with the remaining cases requiring some sort of follow up. In most cases, respondents did not report their client's DTC-GT as 'very useful' in terms of clinical utility $(n=20,80 \%)$, although a large number of respondents reported their client's DTC-GT as 'somewhat useful' ( $n=11,44 \%)$. There was no clear relationship between perception of the DTC-GT process and outcome of consultation. No respondents perceived discussing DTC-GT with their client(s) in a clinical consultation to be beneficial or anxiety-creating for themselves.

\section{Genetic health professionals' perception of client experiences}

Exploratory analysis using cross tabulation of Part 2 data identified possible associations, although the numbers were too small for any statistical testing. Most cases where clients' motivation for testing was to identify monogenic conditions $(n=9)$ were considered to be both beneficial $(n=6,67 \%)$ and anxiety-creating $(n=8,89 \%)$ for the client. Cases where clients' motivation for testing was to know their susceptibility or predisposition for complex conditions $(n=14)$ were rarely considered beneficial $(n=1,7 \%)$ and often considered anxiety-creating $(n=10,72 \%)$ for the client. Although there was no apparent association between client experience and satisfaction level, those clients not satisfied with their DTC-GT $(n=9)$ were likely to have been seeking 
Table 3 Clients' perceived opinions and experiences with DTC-GT

\begin{tabular}{|c|c|}
\hline & $\begin{array}{c}\text { Client, } \mathrm{n}=2 \\
\mathrm{n}(\%)\end{array}$ \\
\hline \multicolumn{2}{|l|}{ Type of DTC-GT ordered } \\
\hline Testing of multiple SNPs or CNVs in multiple genes & $10(40)$ \\
\hline Whole exome sequencing (next generation sequencing) & $4(16)$ \\
\hline Whole genome sequencing (next generation sequencing) & $4(16)$ \\
\hline Full sequencing of two or more genes & $3(12)$ \\
\hline Full sequencing of a single gene & $2(8)$ \\
\hline Testing of one or more SNPs or CNVs in a single gene & $1(4)$ \\
\hline Unknown & $1(4)$ \\
\hline \multicolumn{2}{|l|}{ Motivation for having DTC-GT ( $\geq 1$ motivation per client) } \\
\hline To know their susceptibility/predisposition for complex conditions & $14(56)$ \\
\hline To identify monogenic conditions, including carrier testing & $9(36)$ \\
\hline For academic purposes (eg, interest or curiosity) & $3(12)$ \\
\hline $\begin{array}{l}\text { For other non health-related applications (eg, ancestry, } \\
\text { nutrigenetics, paternity testing) }\end{array}$ & $1(4)$ \\
\hline For pharmacogenetic uses & $0(0)$ \\
\hline To assess their sporting ability & $0(0)$ \\
\hline \multicolumn{2}{|l|}{$\begin{array}{l}\text { Reasons given for seeking professional services in regard } \\
\text { to their DTC-GT ( } \geq 1 \text { reason per client) }\end{array}$} \\
\hline Needed help interpreting the results & $20(80)$ \\
\hline Concerned for health of family members & $15(60)$ \\
\hline Seeking reproductive risk information & $11(44)$ \\
\hline Concerned for own health & $10(40)$ \\
\hline Seeking information on specific conditions & $10(40)$ \\
\hline Seeking further genetic testing & $4(16)$ \\
\hline Questioning validity of DTC-GT results & $2(8)$ \\
\hline \multicolumn{2}{|l|}{ Satisfaction with chosen DTC-GT service } \\
\hline Very satisfied & $8(32)$ \\
\hline Somewhat satisfied & $8(32)$ \\
\hline Not satisfied & $9(36)$ \\
\hline \multicolumn{2}{|l|}{ Feelings of regret for having DTC-GT } \\
\hline No & $14(56)$ \\
\hline Yes & $5(20)$ \\
\hline Unknown & $6(24)$ \\
\hline
\end{tabular}

Abbreviations: CNV, copy number variation; DTC-GT, direct-to-consumer genetic testing; SNP, single-nucleotide polymorphism.

information regarding their susceptibility or predisposition for complex conditions $(n=8,89 \%)$.

\section{DISCUSSION}

This is the first reported survey of genetic health professionals in Australia and New Zealand related to DTC-GT. The reported frequency of individuals presenting to genetics services after having undertaken DTC-GT was $11 \%$ in this study. Further action followed the initial consultation in the majority of cases. Respondents described all clients as concerned for the health of either themselves or family members. They also reported that the majority of clients needed help interpreting DTC-GT results $(80 \%)$; however, in general were not seeking further genetic testing (84\%).

A third of genetic counsellors and clinical geneticists selected 'possibly' in answer to opinion-based questions in this survey, indicating that they do not have firm opinions regarding DTC-GT. The differences in opinion noted between professions may reflect their
Table 4 Genetic health professionals' opinions and experiences regarding DTC-GT in their clinical practice

\begin{tabular}{lc}
\hline & Client, $\mathrm{n}=25$ \\
& $\mathrm{n}(\%)$ \\
\hline Perception of the DTC-GT process ( $\geq 1$ answer per client) \\
Created anxiety for the client & $19(76)$ \\
Difficult to counsel & $12(48)$ \\
A waste of client time & $9(36)$ \\
Beneficial for the client & $9(36)$ \\
A waste of clinic time & $7(28)$ \\
Easy to manage & $7(28)$ \\
& \\
Outcome of consultation ( $\geq 1$ outcome per client) & $9(36)$ \\
Extensive counselling/multiple consults & $7(28)$ \\
Genetic tests ordered in addition to DTC-GT & $7(28)$ \\
No further action & $4(16)$ \\
Family member(s) referred for genetic consultation/counselling & $2(8)$ \\
Client(s) referred to specialist health professional(s) & $1(4)$ \\
Genetic tests ordered to confirm DTC-GT results & \\
Clinical utility of the DTC-GT & $2(8)$ \\
Very useful & $9(34)$ \\
Somewhat useful & \\
Not useful & \\
\hline
\end{tabular}

Abbreviation: DTC-GT, direct-to-consumer genetic testing.

specific training. In Australasia, training of genetic counsellors involves an emphasis on psychosocial aspects of genetic testing and equity of access. We speculate that clinical geneticists may apply a more clinical approach to genetic testing and therefore be less likely to consider DTC-GT useful for individuals who want anonymous testing and/or are geographically isolated. Few respondents were confident in accurately interpreting and explaining DTC-GT results. This lack of confidence may reflect the lack of experience reported by respondents. The authors also speculate that the level of confidence might change over time as the amount of experience increases.

The opinions of genetic counsellors reported in our study reflect those of their counterparts in the USA's National Society of Genetic Counselors (NSGC) who responded to a survey in early 2008. Over half $(57 \%)$ of the responding NSGC members were uncertain whether they would refer an individual to DTC-GT for anonymous testing and $24 \%$ would not refer for this reason. ${ }^{21}$ Similarly, $42 \%$ of ASGC respondents were unsure whether DTC-GT was useful for individuals who want anonymous testing, whereas another $48 \%$ reported they considered it not useful. In the case of individuals who cannot obtain genetic services due to geographic constraints, $60 \%$ of NSGC respondents were uncertain whether they would refer individuals to DTC-GT and $10 \%$ would not refer for this reason. ${ }^{21}$ ASGC respondents were more decisive in this case with $54 \%$ considering DTC-GT not useful for individuals who are geographically isolated and only $37 \%$ not reporting a firm opinion on this point. More than half of the respondents from both the NSGC $(56 \%)^{21}$ and ASGC (53\%) agreed that DTC-GT is an acceptable method of obtaining genetic testing when genetic counselling is provided.

Although the methodologies and contexts are different, data from previous studies indicate that the proportion of genetic health professionals with DTC-GT experience in their clinical practice may be increasing over time. Six percent of clinical geneticists in the Kanagawa Prefecture of Japan who responded to a survey in late 2007 reported having had a patient seek consultation in regards to 
DTC-GT. ${ }^{22}$ Overall, $14 \%$ of NSGC respondents to the aforementioned survey in 2008 reported they had received requests for DTC-GT interpretation or discussion. ${ }^{21}$ In early 2009 , another survey of health professionals in the USA, of which $91 \%$ were genetic counsellors and the remaining $9 \%$ included medical geneticists, found that $16.5 \%$ had consulted with clients who had undertaken DTC-GT. ${ }^{20}$ We have found the cumulative total in Australia and New Zealand in mid 2011 to be $11 \%$, and responses indicate an increasing trend over time within these countries.

Respondents reported that most consumers (92\%) presenting to genetics clinics were not questioning the validity of their DTC-GT results. One interpretation is that the disclaimers made by DTC-GT companies, that their services should not be used for medical decision making, are not read or comprehended by consumers before they provide their consent. The low frequencies of consumers questioning the validity of their DTC-GT also contrasts with the significant concern in the scientific literature regarding the clinical validity of DTC-GT. ${ }^{27,28}$ However, consumers may face difficulties in obtaining and/or comprehending peer-reviewed literature. Once again, this may reflect the level of informed consent, or lack thereof, being provided by consumers. Respondents reported that most consumers (84\%) were not seeking further genetic testing, a finding in concordance with a study in the USA that reported $9 \%$ of clients had further genetic testing ordered as a result of their consultation. ${ }^{20}$

Questions remain with regard to consumers who may not present to genetics clinics for interpretation of DTC-GT results. Do these consumers question the clinical utility of results, and what is the physical/psychological impact on them? It is possible that some association exists between DTC-GT-induced anxiety and presentation to clinical genetics services, such that individuals who do not seek the assistance of genetic health professionals have no long-term negative psychological effects. In our study, over half of the clients did not show apparent feelings of regret for having DTC-GT (56\%) and were somewhat or very satisfied with their chosen DTC-GT service (64\%). However, the long-term impact of DTC-GT on consumers, both physically and psychologically, is not yet well understood.

The ability to accurately calculate the response fraction is a limitation of this study. Further questions remain with regard to those genetic health professionals who did not respond to the survey. Have they consulted with clients in regard to DTC-GT? If so, what was their experience? Our response fraction $(44 \%)$ is comparable to those reported by similar studies in Japan and the USA. ${ }^{20-22}$ Figures provided by the ASGC and AACG were used to calculate response fractions for our study. The AACG provided current figures for the number of qualified clinical geneticists and trainees in Australia and New Zealand. However, the ASGC provided the total number of registered members, including individuals with a special interest in genetic counselling who may not be qualified in the profession. Hence, the response fraction for genetic counsellors, and therefore the overall response fraction, may in fact be higher than reported.

A further limitation of this study is the possibility of non-response bias, despite the relatively high response fraction. This may be compounded by any bias resulting from the $18 \%$ of respondents who are not currently practising, as it is not known if these individuals were practising during the time period relevant to this study. Exploration of opinions regarding DTC-GT was limited by the large number of 'possibly' responses and inability to specify responses with regard to the type of test. A more extensive survey, with a five point Likert scale for responses and specification of opinion per test type, may gain further information. Additionally, respondents' retrospective client descriptions may not be a true reflection of clients' opinions and experiences. Finally, the wide variety of genetic tests marketed directto-consumer complicates efforts to define health-related DTC-GT, posing a potential limitation to this study. The 25 client descriptions extracted from Part 2 responses were therefore carefully reviewed to avoid inclusion of clients who had not undertaken relevant DTC-GT and eliminate the effect of this limitation on final data.

Future considerations include the long-term impact of DTC-GT on consumers, both physically and psychologically. To address these questions, it would be necessary to identify consumers who have undertaken DTC-GT in order for them to participate in a research study. This could provide the opportunity to identify the uptake of DTC-GT within the general population and arrange a prospective study assessing the long-term behavioural and psychological responses to undergoing DTC-GT. However, identifying these consumers may be difficult. One possible method would be to approach health professionals who could recruit appropriate clients during consultations. Another method would be to recruit consumers through local social networking sites. The low incidence reported here suggests this method might produce a low yield of participants over time, and therefore it may be more efficient to approach DTC-GT companies as a source of participant recruitment.

Identification of the present role that genetic health professionals have in DTC-GT may guide preparation of appropriate guidelines and therefore better management of future cases. Further studies could also focus on the uptake of DTC-GT within the general population and examine consumers' long-term behavioural and psychological response to undergoing DTC-GT. Information from the exploratory analysis of experiences and outcomes is considered hypothesis generating and could therefore form the basis of larger studies. In conclusion, DTCGT does not currently represent a significant cause for referral to genetics services in Australia and New Zealand and the majority of genetic health professionals in these countries lack confidence in being able to accurately interpret and explain DTC-GT results.

\section{CONFLICT OF INTEREST}

The authors declare no conflict of interest.

\section{ACKNOWLEDGEMENTS}

This study was completed in partial fulfilment of the requirements for the Master of Genetic Counselling, University of Melbourne, Victoria, Australia and was supported by the Victorian Government's Operational Infrastructure Support Program. JH is funded by a National Health and Medical Research Council Senior Research Fellowship (436904). We would like to thank the HGSA and ASGC for facilitating recruitment and the genetic health professionals who kindly completed the survey.

1 Geransar R, Einsiedel E: Evaluating online direct-to-consumer marketing of genetic tests: Informed choices or buyers beware? Genet Test 2008; 12: 13-23.

2 Field A, Krokosky A, Terry SF: Direct-to-consumer marketing of genetic tests: Access does not reflect clinical utility. Genet Test Mol Biomarkers 2010; 14: 731-732.

3 Janssens AC, van Duijn C: An epidemiological perspective on the future of direct-toconsumer personal genome testing. Invest Genet 2010; 1: 10.

4 Bloss CS, Schork NJ, Topol EJ: Effect of Direct-to-Consumer Genomewide Profiling to Assess Disease Risk. N Engl J Med 2011; 364: 524-534.

5 Borry P, Howard H, Sénécal K, Avard D: Health-related direct-to-consumer genetic testing: a review of companies' policies with regard to genetic testing in minors. Familial Cancer 2010; 9: 51-59.

6 Howard HC, Avard D, Borry P: Are the kids really all right? Direct-to-consumer genetic testing in children: are company policies clashing with professional norms? Eur J Hum Genet 2011; 19: 1122-1126.

7 Lee SS-J, Crawley L: Research 2.0: Social Networking and Direct-To-Consumer (DTC) Genomics. Am J Bioeth 2009; 9: 35-44.

8 Resnik DB: Direct-to-consumer genomics, social networking, and confidentiality. Am J Bioeth 2009; 9: 45-46. 
9 Wasson K: Direct-to-consumer genomics and research ethics: Should a more robust informed consent process be included? Am J Bioeth 2009; 9: 56-58.

10 Ashley EA, Butte AJ, Wheeler MT et al: Clinical assessment incorporating a personal genome. Lancet 2010; 375: 1525-1535.

11 Roberts M, Riegert-Johnson D, Thomas B: Self diagnosis of Lynch Syndrome using direct to consumer genetic testing: A case study. J Genet Couns 2011; 20: 327-329.

12 Saunders K, Nazareth S, Pressman P: BRCA in the Ashkenazi population: Are current testing guidelines too exclusive? Hered Cancer Clin Pract 2011; 9: 3.

13 Imai K, Kricka LJ, Fortina P: Concordance study of 3 direct-to-consumer genetictesting services. Clin Chem 2011; 57: 518-521.

14 Kutz G: Direct-to-Consumer Genetic Tests: Misleading Test Results are Further Complicated by Deceptive Marketing and Other Questionable Practices. United States Government Accountability Office, 2010

15 Richards M: Reading the runes of my genome: a personal exploration of retail genetics. New Genet Soc 2010; 29: 291-310.

16 Gollust SE, Gordon ES, Zayac C et al: Motivations and perceptions of early adopters of personalized genomics: Perspectives from research participants. Public health genomics 2011; 15: 22-30.

17 Graves KD, Peshkin BN, Luta G, Tuong W, Schwartz MD: Interest in genetic testing for modest changes in breast cancer risk: Implications for SNP testing. Public Health Genomics 2011; 14: 178-189.

18 McGuire AL, Diaz CM, Wang T, Hilsenbeck SG: Social Networkers' Attitudes Toward Direct-to-Consumer Personal Genome Testing. Am J Bioeth 2009; 9: 3-10.
19 Wilde A, Meiser B, Mitchell PB, Schofield PR: Public interest in predictive genetic testing, including direct-to-consumer testing, for susceptibility to major depression: preliminary findings. Eur J Hum Genet 2010; 18: 47-51.

20 Giovanni MA, Fickie MR, Lehmann LS et al: Health-care referrals from direct-toconsumer genetic testing. Genet Test Mol Biomarkers 2010; 14: 817-819.

21 Hock KT, Christensen KD, Yashar BM, Roberts JS, Gollust SE, UhImann WR: Direct-toconsumer genetic testing: an assessment of genetic counselors' knowledge and beliefs. Genet Med 2011; 13: 325-332.

22 Ohata T, Tsuchiya A, Watanabe M, Sumida T, Takada F: Physicians' opinion for 'new' genetic testing in Japan. J Hum Genet 2009; 54: 203-208.

23 Kolor K, Liu T, St Pierre J, Khoury MJ: Health care provider and consumer awareness, perceptions, and use of direct-to-consumer personal genomic tests, United States, 2008. Genet Med 2009; 11: 595.

24 Powell K, Cogswell W, Christianson C et al: Primary care physicians' awareness, experience and opinions of direct-to-consumer genetic testing. J Genet Counseling $2011 ; 1-14$.

25 Bloss CS, Ornowski L, Silver E et al: Consumer perceptions of direct-to-consumer personalized genomic risk assessments. Genet Med 2010; 12: 556-566.

26 Wright CF, Gregory-Jones S: Size of the direct-to-consumer genomic testing market. Genet Med 2010; 12: 594.

27 Roukos DH: Innovation versus evidence: to trust direct-to-consumer personal genomic tests? Expert Rev Mol Diagn 2011; 11: 1-4.

28 Murray ABV, Carson MJ, Morris CA, Beckwith J: Illusions of scientific legitimacy: misrepresented science in the direct-to-consumer genetic-testing marketplace. Trends Genet 2010; 26: 459-461.

Supplementary Information accompanies the paper on European Journal of Human Genetics website (http://www.nature.com/ejhg) 